\title{
Long Term Hypopharyngeal Dysfunction in a Pediatric Patient after Toxic Epidermal Necrolysis: A Case Report and Literature Review
}

\section{Lauren K Reckley ${ }^{*}$, Anthony M Tolisano and Benjamin B Cable}

Department of Otolaryngology, Tripler Army Medical Center, Honolulu, HI, USA

"Corresponding author: Lauren K Reckley, Department of Otolaryngology, Tripler Army Medical Center, Honolulu, HI, USA, Tel: 808-433-3181; Fax: 808-433-9033; Email: lauren.k.reckley.mil@mail.mil

Rec date: Apr 11, 2016; Acc date: Jun 25, 2016; Pub date: Jun 30, 2016

Copyright: ( 2016 Reckley LK, et al. This is an open-access article distributed under the terms of the Creative Commons Attribution License, which permits unrestricted use, distribution, and reproduction in any medium, provided the original author and source are credited.

\begin{abstract}
We report the case of an 8-year-old male who experienced failure to thrive and dysphagia following idiopathic Toxic Epidermal Necrolysis (TEN). Despite a normal barium esophagram preoperatively, a significant degree of hypopharyngeal scarring was noted during direct laryngoscopy and bronchoscopy one year after initial presentation. Hypopharyngeal dysfunction following TEN has been reported in adults. However, there are no cases in the published literature examining this phenomenon in a pediatric patient. The purpose of this report is to highlight the potentially significant aerodigestive tract abnormalities following TEN in a pediatric patient, and to advocate for early direct laryngoscopy in children with mild symptoms. Additionally, this case demonstrates the need for a multidisciplinary approach to TEN in order to minimize morbidity.
\end{abstract}

Keywords: Toxic epidermal necrolysis; Hypopharyngeal dysfunction; Dysphagia

\section{Introduction}

Toxic epidermal necrolysis (TEN) is a rare, life-threatening condition characterized by extensive skin and mucosal involvement. A lower incidence of 0.5 cases per million person-years is estimated in the pediatric population, when compared to adults [1]. Although the exact etiology remains unclear, medication reactions have been implicated as causative [2]. In the acute phase, treatment is primarily supportive and ideally performed at specialized burn centers, with efforts aimed at minimizing insensible water loss and electrolyte imbalances [3]. Mortality rates are high, ranging between $25-50 \%$ in the adult population [2]. To our knowledge, only two published cases have described long term hypopharyngeal dysfunction after TEN; both were in adult patients $[4,5]$. We present a unique case of severe hypopharyngeal disfigurement in a child one year after treatment for idiopathic TEN.

\section{Case Report}

A previously healthy eight-year-old male was transferred to our institution following the acute management of idiopathic TEN in June 2014. A consult was placed to the speech pathologist for evaluation of difficulty feeding in mid-July. Due to a significant degree of oral mucocutaneous lesions and associated pain, the patient developed oral aversion requiring nasogastric tube (NGT) feeds. Supplemental caloric intake was provided through this route for nearly one month. Prior to discharge, the NGT was discontinued, as the patient was able to tolerate adequate oral intake, but he continued to require a soft diet and high calorie liquid supplementation (PediaSure ${ }^{\oplus}$ ).

One month after discharge from the hospital, the patient was seen in the otolaryngology clinic. The oral mucosa was healed and the patient was tolerating a regular diet. The patient's weight had stabilized, although it is important to note that he had been at $<3 \%$ on the growth chart for height and weight since early childhood.

In October 2014, the patient was admitted briefly for management of pneumonia. By February 2015, concerns were raised the patient was not receiving adequate calories and was found to have lost several pounds. His mother noted increasing difficulty with swallowing bread and rice, although no overt choking or signs of aspiration were elicited. A barium esophagram revealed no evidence for strictures or filling defects (Figure 1).

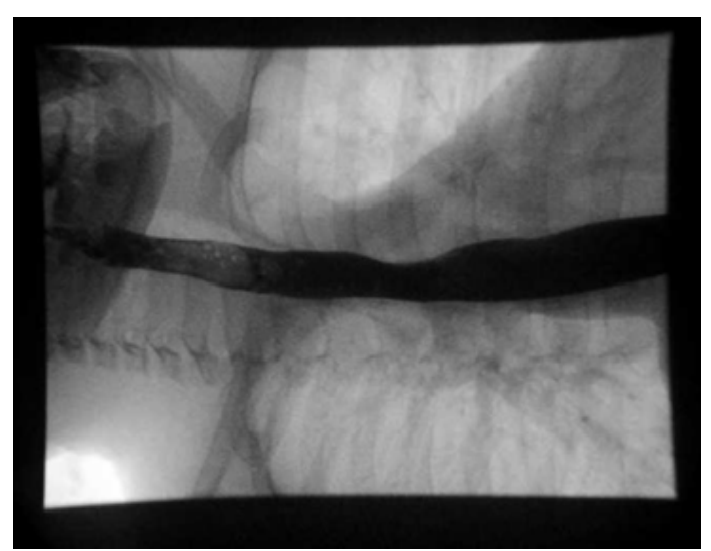

Figure 1: Normal preoperative barium esophagram.

In May 2015, the patient was seen in the multidisciplinary airway clinic for evaluation of chronic productive cough and dysphagia. Given these concerns, the team recommended direct laryngoscopy, bronchoscopy, and esophagoscopy. 
Citation: Reckley LK, Tolisano AM, Cable BB (2016) Long Term Hypopharyngeal Dysfunction in a Pediatric Patient after Toxic Epidermal Necrolysis: A Case Report and Literature Review. J Clin Case Rep 6: 820. doi:10.4172/2165-7920.1000820

Page 2 of 3

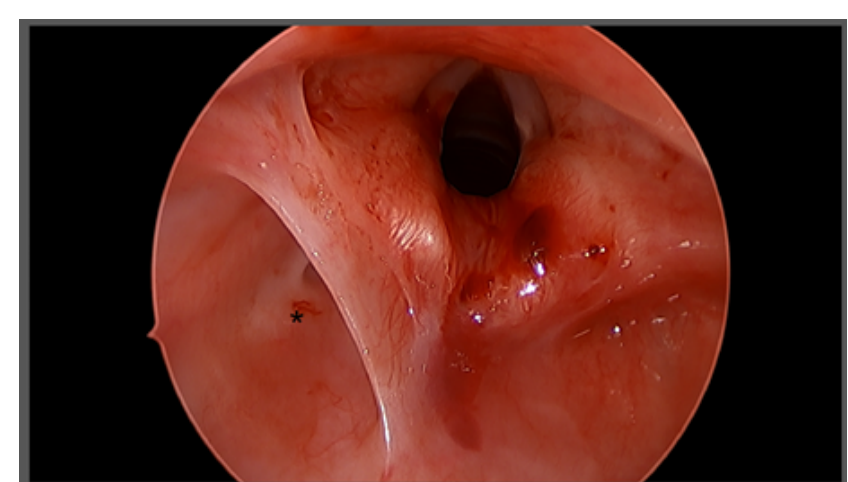

Figure 2: Narrowed esophageal inlet and associated supraglottic scarring (image 020).

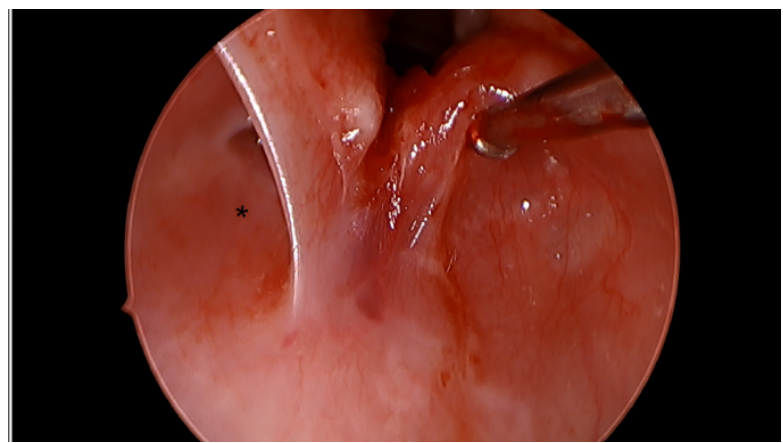

Figure 3: Complete obliteration of the left arytenoid (image 014).

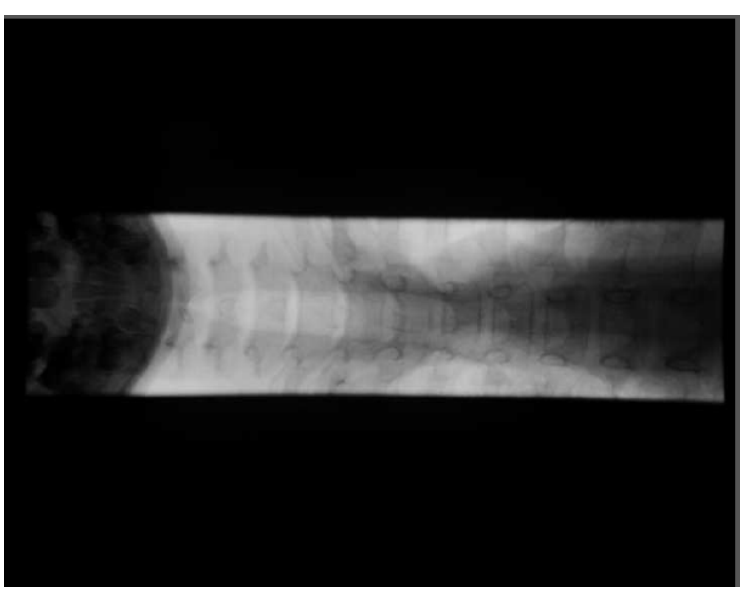

Figure 4: Post-operative modified barium swallow with deviation of barium column to the left.

The patient was taken to the operating room approximately 12 months following his admission for TEN. Direct laryngoscopy revealed scarring of the vallecula and the esophageal inlet, with near complete obliteration of the left arytenoid and supraglottis (Figures 2 and 3).
The distal airways were unremarkable. Esophagoscopy was deferred to avoid the risk of perforation. The patient's post-operative course was uncomplicated. Several days after the procedure, modified barium swallow examination revealed deviation of the barium column to the left following deglutition, which correlated with the intraoperative findings (Figure 4).

\section{Discussion}

Although Stevens-Johnson syndrome and TEN were initially regarded as separate entities, today they are considered variants of the same pathologic process. TEN, the more severe form, is defined by involvement of at least two mucous membranes, loss of confluent sheets of epidermis, and involvement of at least $20 \%$ total body surface area [2]. There is an estimated $9.5 \%$ mortality rate in children suffering from TEN [1]. Historically, Drs. Stevens and Johnson reported two cases of children with fever and associated stomatitis, ophthalmia, and disseminated cutaneous macules with central necrosis [2]. Since that time, the literature has been dominated by adult pathology, as this disease has been found to be far less common in children. Consequently, there is little known about long term sequelae in the pediatric population after acute TEN resolution. Recognition of long term morbidity is essential in survivors in order to maximize quality of life and avoid dysfunction.

As an inpatient with active TEN, our patient was evaluated by both the ophthalmology and dermatology services. Evaluation by the otolaryngology service was not obtained until after the patient had been discharged. In a recent review by Saeed et al, the authors advocate for a multidisciplinary approach to any organ system that will affect the quality of life of TEN survivors [6]. We agree with this recommendation. In the case of rare and systemic disease processes, "widening of the net" to include multiple subspecialists is important in order to avoid missing complications. This is likely to include, among others, otolaryngology, pulmonology, and gastroenterology. A systematic team approach promotes parallel and independent lines of evaluation.

Our case underscores the importance of direct visualization in children with even minor symptoms, as significant abnormalities are possible despite few complaints. In the first few months after treatment of acute TEN, our patient had a clinically normal swallow function. The extent of his swallowing dysfunction remained under appreciated until operative evaluation a year after resolution of acute TEN, despite a completely unremarkable preoperative barium esophagram.

\section{Conclusion}

Toxic Epidermal Necrolysis (TEN) is a rare disease that carries significant mortality and morbidity for both adult and pediatric patients long after resolution of acute disease. Multiple disciplines should be consulted early in the hospital course to minimize long term complications. In this case, a barium esophagram failed to identify the hypopharyngeal scarring that was easily visualized in the operating room. Consequently, a low threshold for direct laryngoscopy is recommended to make accurate diagnoses of stricture and scarring in pediatric patients with TEN.

\section{References}

1. Rizzo JA, Johnson R, Cartie RJ (2015) Pediatric Toxic Epidermal Necrolysis: Experience of a Tertiary Burn Center. Pediatr Dermatol 32: 704-709. 
Citation: Reckley LK, Tolisano AM, Cable BB (2016) Long Term Hypopharyngeal Dysfunction in a Pediatric Patient after Toxic Epidermal Necrolysis: A Case Report and Literature Review. J Clin Case Rep 6: 820. doi:10.4172/2165-7920.1000820

Page 3 of 3

2. Lissia M, Mulas P, Bulla A, Rubino C (2010) Toxic epidermal necrolysis (Lyell's disease). Burns 36: 152-163.

3. Weinand C, Xu W, Perbix W, Lefering R, Maegele M, et al. (2013) 27 years of a single burn centre experience with Stevens-Johnson syndrome and toxic epidermal necrolysis: analysis of mortality risk for causative agents. Burns 39: 1449-1455.

4. Barrera JE, Meyers AD, Hartford EC (1998) Hypopharyngeal stenosis and dysphagia complicating toxic epidermal necrolysis. Arch Otolaryngol Head Neck Surg 124: 1375-1376.
5. Vourexakis Z, Heymans F (2015) Hypopharyngeal Synechiae in Lyell Syndrome (Toxic Epidermal Necrolysis). Otolaryngol Head Neck Surg 153: 152-153.

6. Saeed H, Mantagos IS, Chodosh J (2016) Complications of StevensJohnson syndrome beyond the eye and skin. Burns 42: 20-27.

7. Edell DS, Davidson JJ, Muelenaer AA, Majure M (1992) Unusual manifestation of Stevens-Johnson syndrome involving the respiratory and gastrointestinal tract. Pediatrics 89: 429-432. 